\title{
SOME MEASURE THEORETIC ASPECTS OF STEFFENSEN'S AND REVERSED STEFFENSEN'S INEQUALITY
}

\section{Julije JAKŠEtić, Josip PEČARIĆ AND KSEniJA SMOLJAK KALAMIR}

Abstract. We find necessary and sufficient conditions for Steffensen's and inverse Steffensen's inequality in measure theoretic settings. We also explain natural transition from the Riemann integral in Steffensen's inequality to an integral with measure.

Mathematics subject classification (2010): Primary 26D15; Secondary 26D20.

Keywords and phrases: Steffensen's inequality, reversed Steffensen's inequality, measure theory.

\section{REFERENCES}

[1] P. Billingsley, Probability and Measure, 2nd edn, John Wiley \& Sons, 1986.

[2] J. C. EVARD, H. GAUCHMAN, Steffensen type inequalites over general measure spaces, Analysis 17 (2-3) (1997), 301-322.

[3] A. M. FinK, Steffensen type inequalities, Rocky Mountain J. Math. 12, 4 (1982), 785-793.

[4] H. Gauchman, A Steffensen type inequality, J. Inequal. Pure Appl. Math. 1, 1 (2000), Article 3.

[5] H. Gauchman, On further generalization of Steffensen's inequality, J. Inequal. Appl. 5, 5 (2000), 505-513.

[6] J. JAKŠEtić, J. PEČARIĆ, Steffensen's means, J. Math. Inequal. 2, 4 (2008), 487-498.

[7] J. JAKŠETIĆ, J. PEČARIĆ, Exponential convexity method, J. Convex Anal. 20, 1 (2013), 181-197.

[8] J. JAKŠEtiĆ, J. PEČARIĆ, Steffensen's inequality for positive measures, Math. Inequal. Appl., 18, 2 (2015), 1159-1170.

[9] Z. LiU, More on Steffensen type inequalities, Soochow J. Math. 31 (3) (2005), 429-439.

[10] G. Milovanović, J. PeČARić, The Steffensen inequality for convex function of order $n$, Univ. Beograd Publ. Elektrotehn. Fak. Ser. Mat. Fiz. 634-677 (1979), 97-100.

[11] D. S. Mitrinović, The Steffensen inequality, Univ. Beograd. Publ. Elektrotehn. Fak. Ser. Mat. Fiz. 247-273 (1969), 1-14.

[12] J. E. PEČARIĆ, Inverse of Steffensen's inequality, Glas. Mat. Ser. III 17 (37) (1982), 265-270.

[13] J. E. PeČarić, F. Proschan, Y. L. Tong, Convex functions, partial orderings, and statistical applications, Mathematics in science and engineering 187, Academic Press Inc., Boston, 1992.

[14] J. PeČArić, K. Smoljak Kalamir, S. Varošanec, Steffensen's and Related Inequalities, A Comprehensive Survey and Recent Advances, Monographs in inequalities 7, Element, Zagreb, 2014.

[15] J. F. STEFFENSEN, On certain inequalities between mean values and their application to actuarial problems, Skand. Aktuarietids. (1918), 82-97. 